\title{
Sequence of the mouse $Q 4$ class I gene and characterization of the gene product
}

\author{
Peter J. Robinson ${ }^{1}$, Dorian Bevec ${ }^{2}$, Andrew L. Mellor ${ }^{3}$, and Elisabeth H. Weiss ${ }^{2}$ \\ ${ }^{1}$ Department of Immunology, German Cancer Research Center, Postfach 101949, D-6900 Heidelberg, Federal Republic of Germany \\ 2 Institute of Immunology, University of Munich, Goethestr. 31, D-8000 München, Federal Republic of Germany \\ 3 Transplantation Biology Section, Clinical Research Centre, Harrow, HA1 3UJ, United Kingdom
}

\begin{abstract}
The $Q 4$ class I gene has been shown to participate in gene conversion events within the mouse major histocompatibility complex. Its complete genomic nucleotide sequence has been determined. The $5^{\prime}$ half of $Q 4$ resembles $H$-2 genes more strongly than other $Q$ genes. Its $3^{\prime}$ end, in contrast, is $Q$-like and contains a translational stop signal in exon 5 which predicts a polypeptide with an incomplete membrane spanning segment. The presence of two inverted B1 repeats suggests that part of the $Q 4$ gene may be mobile within the genome. Gene transfer experiments have shown that the $Q 4$ gene encodes a $\beta^{2}$-microglobulin associated polypeptide of $M_{\mathrm{r}} 41000$. A similar protein was found in activated mouse spleen cells. The Q4 polypeptide was found to be secreted both by spleen cells and by transfected fibroblasts and was not detectable on the cell surface. Antibody binding and twodimensional gel electrophoresis indicate that the $\mathrm{Q} 4$ molecule is identical to a mouse class I polypeptide, Qb-1, which has been previously described.
\end{abstract}

\section{Introduction}

The class I major histocompatibility complex (MHC) antigens are glycoproteins of relative mass $35000-45000$ which are associated with $\beta_{2}$-microglobulin. In mice, the $\mathrm{H}-2 \mathrm{~K}, \mathrm{D}$ and $\mathrm{L}$ antigens are transmembrane proteins expressed on the surface of nearly all cells and are thought to direct $\mathrm{T}$ lymphocyte immune responses to viral and other cell surface antigens (Ploegh et al. 1981). In contrast, class I molecules encoded in the $Q a$ and $T l a$ regions are expressed in only a few tissues and do not appear to direct immune responses in the same way as H-2 (Flaherty 1981). For this reason, it has been suggested that Qa and TL antigens may be markers of cell differentiation (Harris et al. 1984). In C57BL/10 mice, the $Q a$ region contains 10 class I genes, $Q 1-Q 10$ (Weiss et al. 1984). Three biochemically and immunologically distinct proteins as- sociated with this gene cluster have been described; these are Qa-2, Q10 and Qb-1. Qa-2 is found on the surface of several types of hematopoietic cells and also occurs as a secreted molecule (Michaelson et al. 1981, Robinson 1987, Soloski et al. 1986). Q10 is produced only in the liver and is secreted into the blood (Lew et al. 1986). Qb-1 is synthesized by cells from several lymphoid tissues (Robinson 1985).

It was shown previously that the Qa-2 and Q10 molecules are encoded by $Q 6-Q 10$ (Mellor et al. 1985 , Devlin et al. 1985), but no polypeptide products of $Q 1-Q 5$ have so far been described. Recently, $Q 4$ was shown to be the donor gene for the bm6 gene conversion event in C57BL/6 mice, since it has the bm6-specific nucleotide sequence in exon 3 , which encodes the second protein domain (Geliebter et al. 1986). It has been shown previously that the flanking regions of $Q 4-Q 10$ are highly homologous to those of the $H-2 K$ region genes, supporting the hypothesis that the $H-2 K$ gene pair may have arisen by duplication which involved $Q a-2$ region genes (Weiss et al. 1984). It was therefore necessary to obtain the nucleotide sequence of $Q 4$ in order to understand how such gene conversion events could occur. The fact that the $Q 4$ gene is transcriptionally active (Geliebter et al. 1986) prompted us to identify the corresponding polypeptide. Our results show that $Q 4$ encodes a functional class I polypeptide in contrast to $Q 5$, which carries a translational stop signal in exon 3.

\section{Materials and methods}

Nucleotide sequence determination. The $Q 4$ and $Q 5$ genes were subcloned from cosmid Bm1-2 (Weiss et al. 1984) and their nucleotide sequences determined according to the scheme shown in Figure 1 using procedures already published (Maxam and Gilbert 1977, Sanger et al. 1980). Enzymes were purchased from Boehringer Mannheim or New England Biolabs.

Gene transfer and antigen detection. Cosmid or plasmid DNA (10 $\mu \mathrm{g})$ containing $1 \mu \mathrm{g}$ pAG60neo was transfected into $\mathrm{BALB} / \mathrm{c} 3 \mathrm{~T} 3$ cells using 


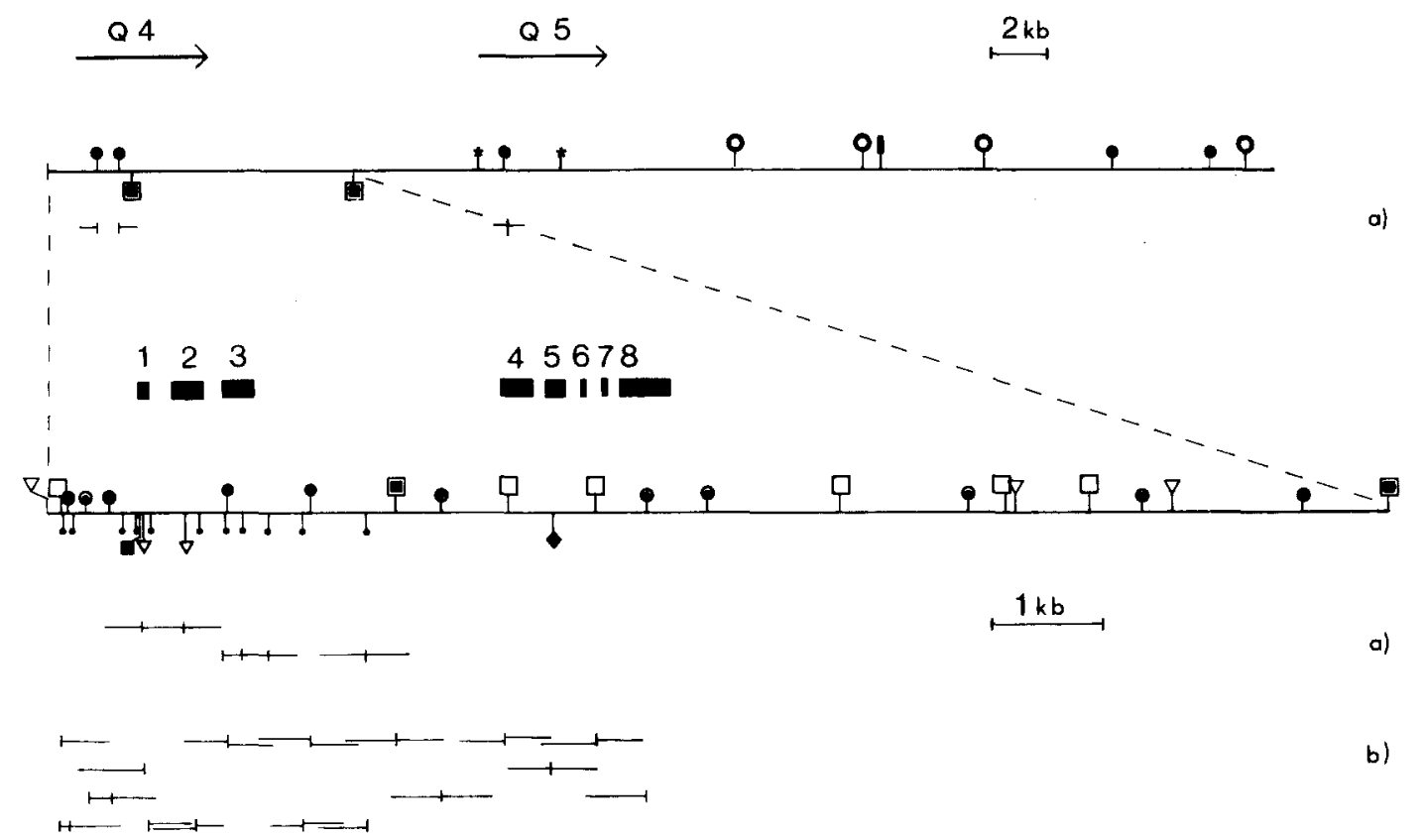

Fig. 1. Restriction maps of cosmid Bm1-2 and the subcloned $Q 4$ gene. Numbered solid boxes represent exons. Restriction site symbols in Bm1-2 are: Cla I, I ; Hpa I, 9 ; Kpn I, $\uparrow$; Sal I, ; and the two Bam HI sites (田) used for subcioning. Restriction site symbols of the $Q 4$ subclone

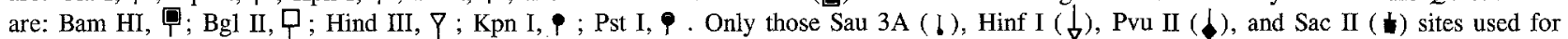
sequencing are shown. (a) Sequences obtained by the chemical method of Maxam and Gilbert (1977). (b) Fragments sequenced by the dideoxy method of Sanger and co-workers (1980)

standard procedures (Wigler et al. 1977). Colonies resistant to $0.8 \mu \mathrm{g}$ G418 were pooled, expanded, and labeled with ${ }^{35} \mathrm{~S}$-methionine as described (Mellor et al. 1985). Mouse spleen cells were cultivated with concanavalin A for $48 \mathrm{~h}$ prior to labeling (Robinson 1987). Class I molecules were detected by immunoprecipitation of cell lysates (Dobberstein et al. 1979) using protein A-sepharose immunosorbents (Robinson 1987). Pulse-chase analysis was performed as described by Dobberstein and co-workers (1979). Surface labeling of cells was performed according to Robinson and Schirrmacher (1979). Two dimensional gels were run as before (Robinson 1985).

Carbonate treatment of microsomes. Microsomal vesicles were isolated from transfectants as described by Dobberstein and co-workers (1979), and treated with sodium carbonate, $\mathrm{pH} 11.5$, as described by Fujiki and co-workers (1982). Soluble and membrane fractions were treated with triton $X-100$ and immunoprecipitates prepared as before.

Molecular weight determinations. To estimate the molecular weights of class I molecules, immunoprecipitates were treated exhaustively with Endo F (Boehringer Mannheim) as described (Robinson 1987), reduced, and separated on $10-15 \%$ polyacrylamide gradient gel slabs using ovalbumin $\left(M_{r} 43000\right)$, glyceraldehyde 3 phosphate dehydrogenase $\left(M_{r}\right.$ $36000)$ and carbonic anhydrase $\left(M_{r} 29000\right)$ as markers. Estimates of molecular weight were obtained by plotting relative migration $\left(R_{f}\right.$ values) against $\log \mathrm{M}_{\mathrm{r}}$. Actual $\mathrm{M}_{\mathrm{r}}$ was calculated from the amino acid sequence.

\section{Results}

The entire $Q 4$ gene is present on two subcloned restriction fragments isolated from cosmid clone Bm1-2 (Weiss et al. 1984). As a result of the cloning procedure, the $5^{\prime}$ end of the gene is present on a $3.1 \mathrm{~kb}$ Hind $\mathrm{III} / \mathrm{Bam} \mathrm{HI}$ fragment and the $3^{\prime}$ end is on a genomic $9 \mathrm{~kb}$ Bam HI fragment. DNA sequence analysis was carried out according to the scheme outlined in Figure 1. The nucleotide sequence of $Q 4$ is shown in Figure 2, together with the partial sequence of $Q 5$. In $Q 4$ no polyadenylation signal is present in the 210 bp of the $3^{\prime}$ untranslated region sequenced.

The promoter region of the $Q 4$ gene. The sequence of the $Q 4$ promoter reveals strong sequence homology to the $5^{\prime}$ flanking sequences of $H-2 K^{b}$ (Kimura et al. 1986) and the $Q$ genes (Devlin et al. 1985). A more detailed inspection shows that, with one exception, the regulatory sequences (e.g., the interferon consensus sequence) are strongly homologous to those of $H-2 K^{b}$. The $Q 4$ gene, however, contains only a mutilated copy of sequence IV, which is part of the $H-2 K^{b}$ enhancer. In $H-2 K^{b}$, this en-

Fig. 2. Sequence of the $Q 4$ gene. Protein translations of the exons are given above the DNA sequence. The untranslated exons 6,7 , and 8 are shown in triplets. The partial sequence of the $Q 5$ gene (exons $2 / 3$ and introns $2 / 3$ ) is written below the Q4 sequence. - denotes nucleotide identity. The interferon consensus sequence, the CCAAT and the TATAAA boxes are indicated with broken lines. Sequences homologous to the B1 repeat, two copies in intron 3 and a portion in the $5^{\prime}$ flanking region, are underlined 
gaICTI IGTITT TAaAaACa GCTGAGAAGACAGATCCTGAGGGAAAAGACGAAG TATCCTCTI TA TAGAGGAGAG TCC TGCAC TCAGGCTT GG CAG TG TGAGCCGCCCAC TGC AGG TGAA CAGAGCCTGGTCTCTGTGG TGTTC TAA TGGGGCT TGCAGGCCAGCGCCIC TGCIT TAAGGAGAAGCC TCICTCCACTGCATCCCCAAGCGCTIG IG TIGC

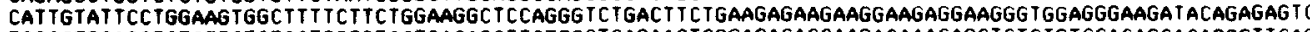
TGGCCTGCAGG TCTCTTCTCTCCTGG TGTCCTGACAGCITCTGGG TCAGAAC T CGGAGACACCAAGACAAACAGCTCTCIGTCCACAGCACAGGG TCAG

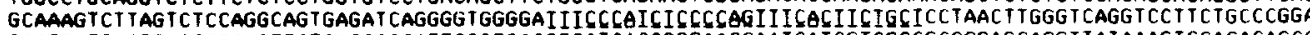
CACTGATGACT TAC TGGCAGT TCTCACCCCCATIGGG TGACG TGATCACCGGGACCCAICATCGTCGCCGCGGGAGCAGG IEIAGAGTCCACACAGCC

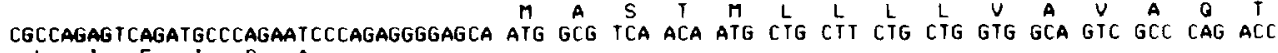

L I E I R A 1 A GCGTCGCCCACCTGACCCICCGCCCCTTCTCCACCCTAGCCCCGCGCCCTGCTCCCCTCCCGGCCCGC TCACCCGCGGGGG TCCCGGAAGGAG TICGGGG

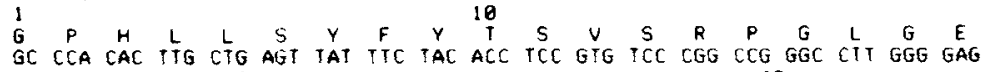

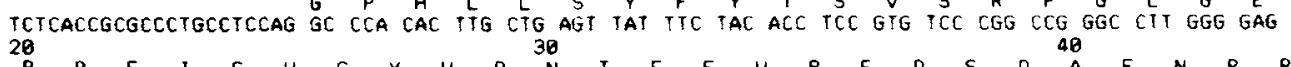

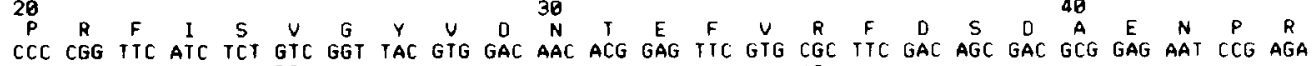
CCC CGG TTC ATC TCT GTC GGT TAC GTG GAC AAC ACG GAG TIC GTG CGC TIC GAC AGC GAC GCG GAG AAT CCG AGA

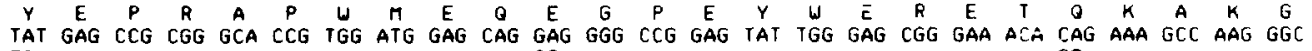
70

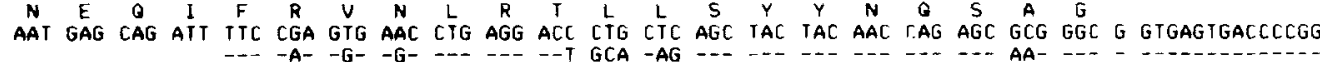

AICGGAGGTCACGA CCCCTCCACGICCCAAAACAGGGGCCCGAGACGTCCCGGGCCCCAAGITCGAGGTICT GAGCAGAACGGACGCGGGACTGGIITC

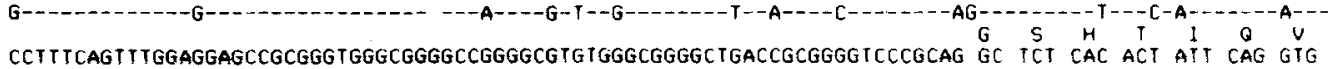
CCIITCAGITIGGAGGAGCCGCGGGTGGGCGGGGCGGGGCGTGTGGGCGGGGCTGACCGCGGGGTCCCGCAG GC TCT CAC ACT ATT CAG GTG

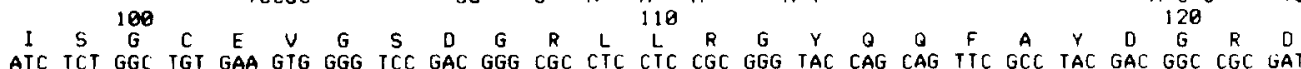
ATC TCT GGC IGT GAA GTG GGG ICC GAC GGG CGC CTC CIC CGC GGG TAC CAG CAG ITC GCC TAC GAC GGC CGC GAT TAC ATC OCC CTG AAC GAA GAC CTG AAA ACG TGG ACG GCG GCG GAC ATG GCG GCA CAG ATC ACC CGA CGC AAG IGG -

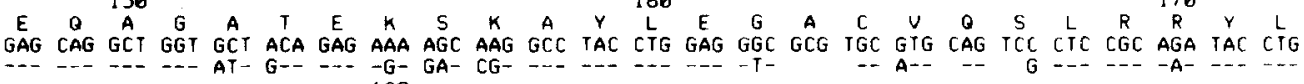

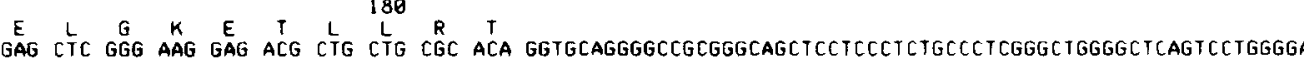
AGAAGAAACCCTCAGCTGGG IGATGCCCCTGTCTCAGAGG GAGAGAGTGACCCTG GTCTCCTGATCCCTCATCACAGTGACTGCACTGACICTCCCAGG GCTCAGCCTICTCCCTGGACAG IGCCCAGGCTGICTCAGGAGGGAAGGAGAGAATITCCTGAGG TAACAACAGCTGCTCCCTICAGTTCCCCTGTAGCC TCTGICAGCCATGGCCTCTCCCAGGCCGGGTICICAGCCCACTGTCIGTAGACAC TGACTCCTGICCTGCTGAG IG TGT CAGCCCITACACCT CAGGACC

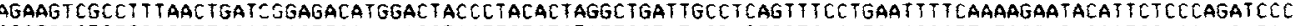
CCC TGTCTG TGGGGTITCCACCCCT ICGACAACCTAATICTCTCTATTCCTATAGTGG TGGTCACATCAGCCCT TATGGGG TACCCTGGAGGAATAICA

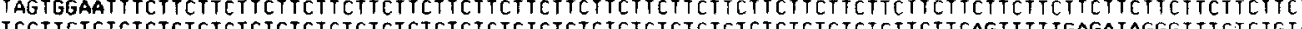
TCCTICTCTCTCTCTCTCTCTCTCTCTCTCTCTCTCTCTCTCTCTCTCTCTCTCTCTCTCTCICTCTCTTCTTCAGT TIT TGAGATAGGG TTTCTCTG TA TGCCCTGGCTGTCC TGGAACTCACT IGTAGACCAGGATGGCCTCGACC T CAGAAATCCGCC TGCCICIGCCTCCCAG TGCIGGGAT I AAAGGCG TGIGGC CACCITGCCCAGCCTITCTTATITTCTTTACTTITTITITTTTTTIGGAGGGG TAATIT TGTTTCTAGTCATCTTTTGICTIT TGTCTGCACTGGAG GATCCTGITTCTCCCTGCCCTTATATTATCATGTGTATCAGTCTCCACAGG TGCCAGGGAAG I TAGACAAG T TAAATCAGGG TCTCIT TAAAGGAGAG

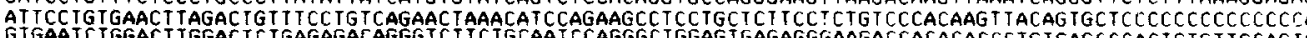

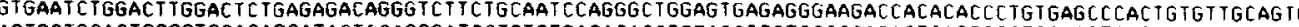
AGIGCTGCACTGGGG CCACAGCATACTCCAGGGATCCTGTGTGACACACC TGTACCI I TGTCCCCCAGAGTCAGGGGCTGGAG TCAT TT TCTC TGGC TG AGTGTCAGAGGT TGACACCATT TCTGCTACACACTCTG TGATGGCTGCTCACT TGGACTGGCGGT TATGCT TAGT TAG CAAGA GAACACAG TGG TIIACG TCTCAG TG TCACACCCT TCCAGTGGCATATGGCTCTAAIT ICIAC TIT IGATACGAACTCAAACACTTAT TAAATTAGT TAGTT TCCAT TCCATCT TCC TGTGCAGCTGAGGAAACCAGCTCTGCCTGCAGGTCACCAG TGCCATGACAG T TGAGG TG T CAAACAGACACAITG T TCAG TGTCATIAGTGAT TTAAC T

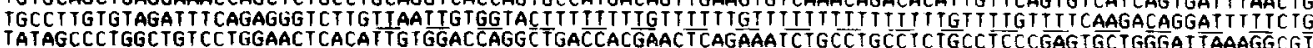

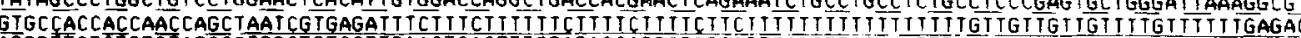

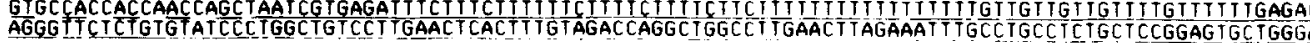
D P $P$ A H 190

TIAAGGGCATg TGCCACTACCAACCAGCTAAITGIGgGaTtICTIAAATCTICCACACAG AT CCT CCA AAG GCA CAT GTG ACA

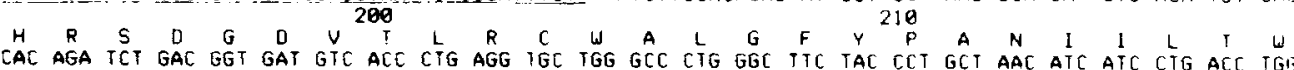
220 G051 $Q$
CAG TTG AAT GGG GAG GAG CTG ACC CAG GAC AIG GAG CIT GTG GAG ACC AGG CCT TCA GGG GAT GGA ACC IIC CAIJ CAG TTG AAT GGG GAG GAG CTG ACC CAG GAC AIG GAG CIT GTG GAG ACC AGG CCT TCA GGG GAT GGA ACC IIC CAIS AAG TGG GCA TCT GIG GTG GTG CCT CIT GGG AAG GAG CAG AAT TAC ACA IGC CAT GTG CAC CAT GAG GGG CTIS CCT E. P $P$ L T L R

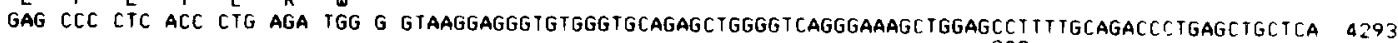
GgGCTGAGAGCTGGGGTCATGACCTCACCTICATTICCTGTACCTGTCCTTCCCAG AG CCT CCT CCA TCC ACT GTC ICL AAC ATG GCG

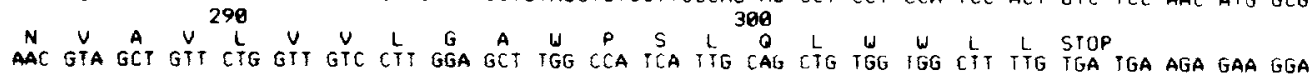

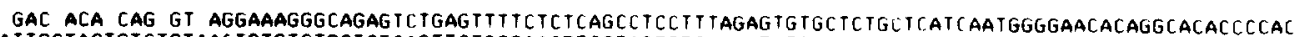
AITGCTACTGTCTGTAAC TGTGTC TGCTCTCAGTTCTGGGAACTICCTAG IG CAAGCTC TICCTIGAACTCTCACAGCTTICCT TCTCACAG GI GGA CAA GGA GGG GAC TAT GCT CTG GCT CCA G GITAGTGTGGGGGACAGAGT TGICCTGAGGTCATTGGAG TGAAGCTGGAGTIGTIGGGTGCT CIGGGAACCCATAATAGCT TCICTGT TGTAATCCTCTGG TGGCCTGTGTCAGAICT TGCTATAGATATATCTT IG TATATATTY TLCCTAGGCAG GG ACA GCT CCC AGA GCT CTG ATA TGT ITC TCT CAA GAT TGI AAA G GTGACATICTATGGCCTGATTGCAGAGGGGCAC TG TGGACAT

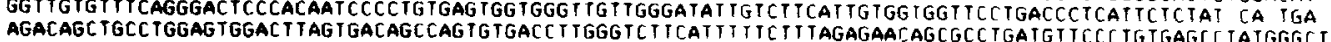

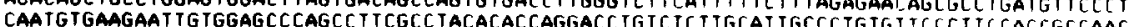


Table 1. DNA sequence homology

\begin{tabular}{|c|c|c|c|c|c|c|c|}
\hline & \multirow{2}{*}{$\begin{array}{l}\text { Length } \\
\text { Q4 }\end{array}$} & \multicolumn{6}{|c|}{ Changes } \\
\hline & & $\mathrm{Q} 4 / \mathrm{K}^{\mathrm{b}}$ & $\mathrm{Q} 4 / \mathrm{D}^{\mathrm{d}}$ & Q4/Q5 & $\mathrm{Q} 4 / \mathrm{Q} 7$ & Q4/Q8 & $\mathrm{Q} 4 / \mathrm{Q} 10$ \\
\hline Exon 1 & 64 & 31.2 & 57.8 & & 14.1 & 14.1 & 29.7 \\
\hline Intron 1 & 195 & 12.3 & 12.8 & & 13.3 & 13.3 & 14.9 \\
\hline Exon 2 & 270 & 8.5 & 9.6 & & 13.3 & 13.3 & 10.7 \\
\hline Intron 2 & 186 & 11.3 & 12.9 & 16.7 & 16.1 & 16.1 & 17.2 \\
\hline Exon 3 & 276 & 8.7 & 10.9 & 11.2 & 8.3 & 9.1 & 11.2 \\
\hline Exon 4 & 276 & 7.2 & 6.1 & & 5.8 & 5.4 & 5.8 \\
\hline Intron 4 & 126 & 3.1 & 6.3 & & 8.7 & 2.4 & 6.3 \\
\hline Exon 5 & 116 & 10.3 & 19.0 & & 10.3 & 3.4 & 15.5 \\
\hline Intron 5 & 178 & 2.8 & 9.5 & & 12.4 & 3.4 & 9.5 \\
\hline Exon 6 & 33 & 0 & 3.0 & & 9.1 & 0 & 12.1 \\
\hline Intron 6 & 163 & 19.6 & 24.0 & & 22.1 & 1.2 & 22.1 \\
\hline Exon 7 & 40 & 12.5 & 5.0 & & 17.5 & 0 & 15.0 \\
\hline Intron 7 & 135 & 14.1 & 15.6 & & 16.3 & 0.7 & 21.5 \\
\hline $3^{\prime} \mathrm{UT}$ & 207 & 15.0 & 20.8 & & 16.4 & 2.4 & 22.2 \\
\hline
\end{tabular}

Nucleotide sequence data were obtained from the following sources: $H-2 K^{b}$, Weiss et al. $1983 ; H-2 D^{d}$, Taylor Sher et al. $1985 ; Q 7, Q 8$, Devlin et al. 1985; $Q 10$, Mellor et al. 1984

hancer element is composed of two overlapping $18 \mathrm{bp}$ repeats, only one copy of which is found in $Q 4$.

Repetitive sequences in the Q4 gene. It is interesting that at the $3^{\prime}$ end of intron 3, the $Q 4$ gene contains two direct, but not exact, $280 \mathrm{bp}$ copies of the B1 repeat (Kalb et al. 1983), as indicated in Figure 2. This element is not found in this position in any other $\mathrm{H}-2$ class I genes. The limited sequence data available shows that this repeat is present in the reverse orientation at the very $5^{\prime}$ ends of $Q 7$ and $H-2 K^{b}$, as well as in $Q 4$ (Steinmetz et al. 1981, Devlin et al. 1985, Kimura et al. 1986). Thus, exons 1, 2, and 3 of $Q 4$ are flanked by inverted DNA sequence repeats which may render the polymorphic $5^{\prime}$ half of the gene mobile within the genome.

Comparison of $Q 4$ with other mouse class I genes. Since no $Q 4$ cDNA clones have been isolated so far, the boundaries of the protein-coding exons of $Q 4$ were assigned using consensus splice-donor and acceptor sequences, as well as by comparison with the $H-2 K^{b}$ gene (Weiss et al. 1983). Thus the regions marked in triplets near the bottom of Figure 2 correspond to exons 6,7 , and 8 and the $3^{\prime}$ untranslated region of $H-2 K^{b}$. Table 1 shows percentage homologies between $Q 4$ and other class I genes. All comparisons are between genes of the $H-2^{b}$ haplotype, with the exception of $H-2 D^{d}$, which was used because only a cDNA sequence is available for $H-2 D^{b}$ (Reyes et al. 1982). The percentage homology was not calculated for intron 3 because multiple insertions and deletions make alignment of the different sequences impossible. For sim- plicity, only comparisons with $Q 4$ are given in Table 1 . Comparisons among other genes in Table 1 have been published elsewhere (Weiss et al. 1983, Mellor et al. 1984, Devlin et al. 1985, Arnold et al. 1984).

The $5^{\prime}$ half of the $Q 4$ gene (e.g., introns 1 through 3 and intron 4) shows particularly strong sequence homology to $H-2$ genes. This level of homology is significantly greater than that normally found for nonallelic class I genes. Particularly strong homology to $\mathrm{H}-2$ genes is found in short stretches, for example in the last $80 \mathrm{bp}$ of intron 2 , the first $147 \mathrm{bp}$ of exon 3 and the first $63 \mathrm{bp}$ of exon 5. In exon 4 , which encodes the conserved third protein domain, $Q 4$ is no more homologous to other class I genes than they are to each other.

Comparison of $Q 4$ with other $Q$ genes. Published comparisons of $Q$ gene sequences suggest that $Q 7$ and $Q 9$ may be pseudo-alleles. In addition, the $5^{\prime}$ half of $Q 8$ is highly homologous to that of its odd-numbered partner, $Q 7$ or $Q 9$ (Devlin et al. 1985). In contrast, the $5^{\prime}$ half of $Q 4$ differs significantly from the corresponding regions of other $Q$ genes. As was mentioned previously, this segment is more closely related to $H-2$ genes (e.g., $H-2 K^{b}$, $H-2 D^{b}$, and $H-2 D^{d}$ ) than it is to other $Q$ region genes. In its $3^{\prime}$ half, starting in intron 3, Q4 is similar to its evennumbered counterpart $Q 8$, and these two genes are virtually identical from intron 5 onwards.

Comparison of the $Q 4$ sequence with other published mouse class I sequences reveals a possible analog in the $\mathrm{BALB} / \mathrm{c}$ mouse. The partial DNA sequence of the genomic clone H-2D28.5 (Jaulin et al. 1985), covering exons 
1,2 , and 3 , shows only three nucleotide differences in the polymorphic exons 2 and 3 . Several differences are found in exon 1, which encodes the signal sequence, as is commonly observed between allelic class I genes. An additional nucleotide in exon 1 of $\mathrm{H}-2 \mathrm{D} 28.5$ may cause a frameshift preventing expression of the polypeptide. Since no restriction map of $\mathrm{H}-2 \mathrm{D} 28.5$ has been published it is difficult to say conclusively whether this gene is allelic with $Q 4$.

The Q5 gene is a pseudogene. The partial nucleotide sequence of the $Q 5$ gene from the cosmid clone Bm1-2 (Fig. 2) covering exon 2 , intron 2 and exon 3 reveals a series of three small deletions in exon 3 resulting in an early translational stop signal at amino acid position 164. A mRNA derived from this gene would direct the synthesis of a short polypeptide lacking the third and subsequent protein domains.

Structure of the $Q 4$ gene product. The nucleotide sequence predicts that $Q 4$ directs the synthesis of a polypeptide which differs significantly from other class I molecules in its third protein domain. In this conserved protein domain, several residues are present which are unique to $Q 4$. In addition, at amino acid 296 in the membrane-spanning domain, a single nucleotide deletion causes a premature translational stop signal after amino acid 305. The same deletion is also found in the $Q 8$ gene (Devlin et al. 1985). Thus, the hydrophobic transmembrane domain of $Q 4$ is incomplete and may be incapable of providing an adequate membrane anchor for the polypeptide chain. To identify the Q4 polypeptide, the cosmid clone B2.5 (Weiss et al. 1984), containing both the $Q 4$ and $Q 5$ genes, was transfected into $\mathrm{BALB} / 3 \mathrm{~T} 3$ fibroblasts using the $\mathrm{Ca}_{2} \mathrm{PO}_{4}$ method (Wigler et al. 1977). In the same experiment, $3 \mathrm{~T} 3$ cells were transfected with two other cosmid clones, B1.24 and $\mathrm{H} 26$, containing $Q 1-Q 3$. In addition, a plasmid subclone of the $Q 4$ gene on a $10 \mathrm{~kb}$ Eco RI fragment was also tested. Transfectants were labeled biosynthetically with a short pulse of ${ }^{35} \mathrm{~S}$-methionine and class I molecules were detected by immunoprecipitation of cell lysates with the rat monoclonal antibody, R1.9.6, which recognizes determinants common to several mouse class I molecules. Immunoprecipitates were analyzed by oneor two-dimensional gel electrophoresis. As shown in Figure 3a, 3T3 cells transfected either with cosmid B2.5 or with the Q4 subclone synthesized a polypeptide of $\mathrm{M}_{\mathrm{r}}$ 41000 which was not present in cells transfected with B1.24 or H26. A polypeptide of similar size was detected in lysates of ${ }^{35} \mathrm{~S}$-methionine labeled C57BL/6 spleen cells. The polypeptides of $M_{r} 43000$ and 45000 present in all $3 T 3$ lysates are $H-2 D^{d}$ and $H-2 L^{d}$ respectively, both of which react with $\mathrm{R} 1.9 .6$. In C57BL/6 spleen cell lysates, however, R1.9.6 detects only the Q4 polypeptide. Due to the co-precipitation of $\mathrm{H}-2$ molecules by $\mathrm{R} 1.9 .6$, it was not possible to obtain all the necessary biochemical data on Q4 using the transfectants alone and therefore spleen cells were used for some experiments. Two-dimensional gel analysis of class I material isolated from transfectants shows that the Q4 polypeptide is a homogeneous species which is smaller and more basic than the H-2D and $\mathrm{H}-2 \mathrm{~L}^{d}$ antigens from the host cell line (Fig. 3b). No polypeptides attributable to the $Q 1, Q 2, Q 3$, or $Q 5$ genes have so far been detected.

Biosynthesis of the Q4 polypeptide. Pulse-chase analysis was used to follow the biosynthesis of the Q4 polypeptide in transfected $3 \mathrm{~T} 3$ fibroblasts and in C57BL/6 spleen

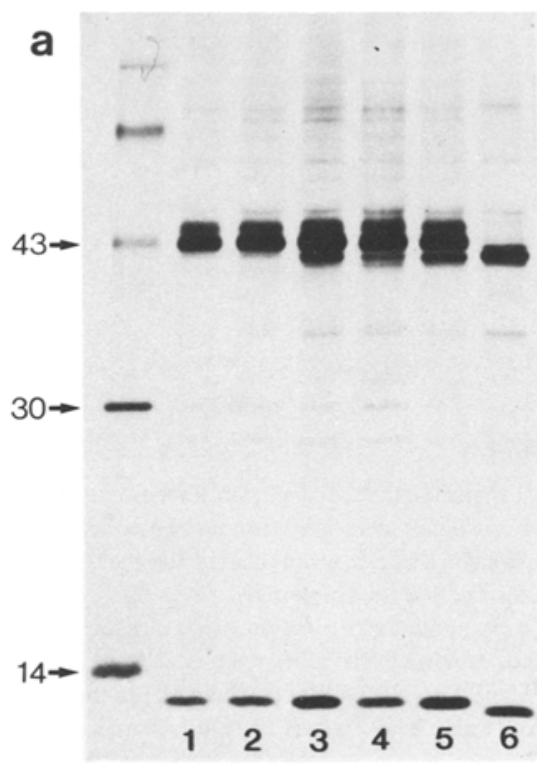

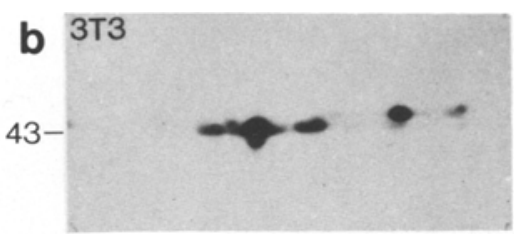
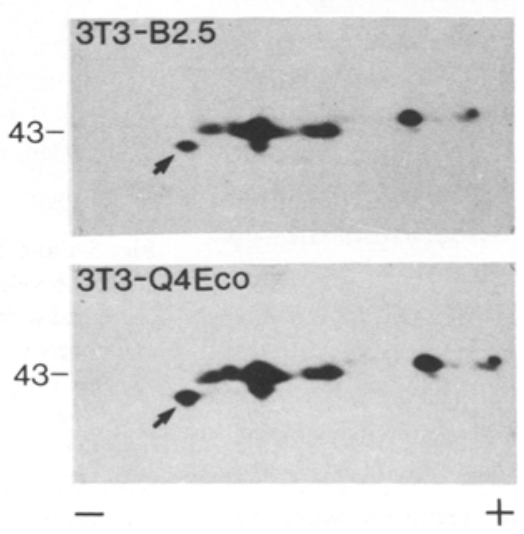

Fig. 3. Fluorograms of one- and twodimensional polyacrylamide gels showing synthesis of ${ }^{35} \mathrm{~S}$-methionine labeled Q4 polypeptides. (a) $10-15 \%$ gradient SDS gel showing polypeptides precipitated from transfected $3 \mathrm{~T} 3$ cells (lanes 1-5) and C57BL/6 spleen cells (lane 6) using antibody R1.9.6. DNAs transfected were cosmids B1.24 ( $Q 1$, lane 1$)$, H26 $(Q 2+Q 3$, lane 2$)$, B.2.5 $(Q 4+Q 5$, lanes 3 and 4 ), and a $Q 4$ Eco RI subclone (lane 5). (b) Two-dimensional gels of class I molecules synthesized by untransfected or transfected 3T3 cells and precipitated with R1.9.6. Arrows indicate Q4 polypeptides. Markers at border are kilodaltons and indicate running positions of ${ }^{14} \mathrm{C}$-labeled marker proteins. Fluorograms were exposed for 3 days (a) or 7 days (b) using Kodak XAR-5 film 


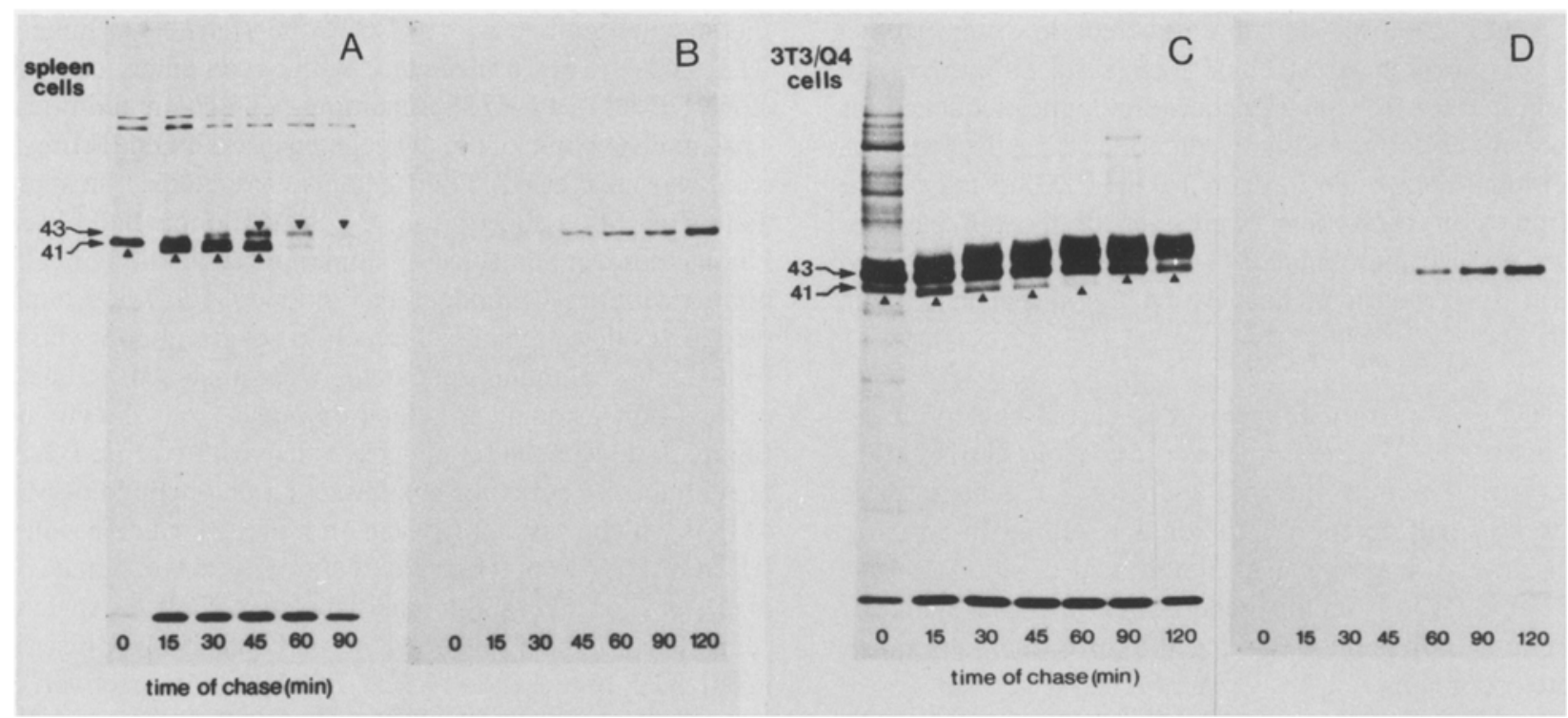

Fig. 4. Processing and secretion of Q4 polypeptides. C57BL/6 spleen cells (panels a and b) or 3T3 cells transfected with Q4 Eco RI DNA (panels $\mathrm{c}$ and d) were labeled for $5 \mathrm{~min}$ with ${ }^{35} \mathrm{~S}$-methionine and chased for the times shown. Panels a and c show immunoprecipitates of cell lysates and panels $\mathrm{b}$ and $\mathrm{d}$ are from the corresponding supernates, using R1.9.6 antibody. Conditions as for Figure 3a. Pointers indicate running position of Q4 polypeptides

cells. Cells were labeled with a short pulse of ${ }^{35} \mathrm{~S}-$ methionine and chased for various times in complete medium. Both cells and supernates were treated with R1.9.6 to precipitate class I polypeptides. As shown in Figure 4, Q4 molecules, in contrast to $\mathrm{H}-2$ antigens, undergo a small but characteristic reduction in $\mathrm{M}_{\mathrm{r}}$ after about $15 \mathrm{~min}$ of chase. This shift was not observed if the samples were treated with endoglycosidase F (Elder and Alexander 1982) prior to electrophoresis, indicating that modification of $\mathrm{N}$-linked carbohydrates is responsible (data not shown). A similar shift was observed in Q4 polypeptides derived from C57BL/6 spleen cells (Fig. 4). The processing of $\mathrm{Q} 4$ precursors to their corresponding mature forms could best be followed in spleen cells, since in transfectants the mature forms are masked by $\mathrm{H}-2$ molecules. In spleen cells, Q4 precursor polypeptides are processed to $M_{r} 43000$ forms which diminish in quantity during the chase period. After about 45 min of chase, soluble class I molecules of $M_{r} 43000$ were detected in the supernates of spleen cells and fibroblasts expressing the Q4 gene, but not in supernates of control transfectants. Thus, both transfected fibroblasts and spleen cells synthesize Q4 polypeptides which are processed within the cell and are secreted into the culture medium.

It was shown recently that Qa-2 exists both as a membrane-associated and as a secreted molecule (Robinson 1987, Soloski et al. 1986). The data suggest that soluble Qa-2 is derived from the membrane form by processing. To determine whether secreted Q4 was derived from a membrane-associated precursor, transfectants were la-
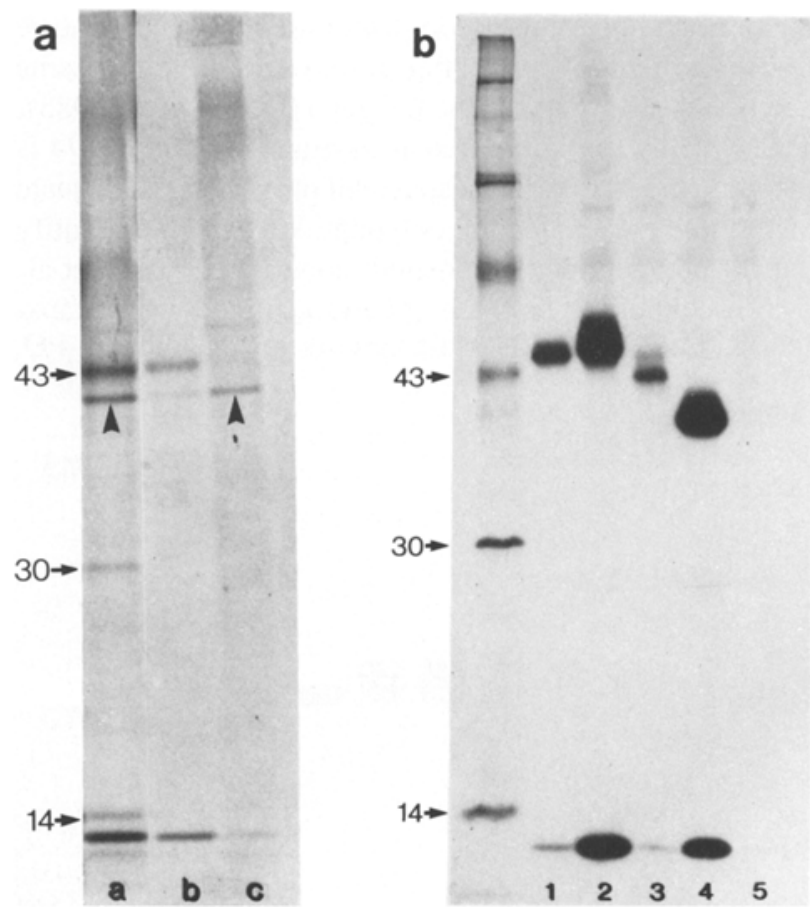

Fig. 5A and B. A Class I molecules associated with microsomes from C57BL/6 spleen cells. Microsomes were used unfractionated (lane a), or treated with sodium carbonate $\mathrm{pH}$ 11.5. Lanes $\mathrm{b}$ and $\mathrm{c}$ show molecules associated with the insoluble and soluble fractions respectively. Pointers indicate Q4 polypeptides. B Immunoprecipitation of class I molecules from lysates of ${ }^{125} \mathrm{I}$-surface-labeled C57BL/6 spleen cells. Antibodies used were $9.178\left(\mathrm{H}-2 \mathrm{~K}^{\mathrm{b}}\right.$ lane 1$), \mathrm{B} 22.249\left(\mathrm{H}-2 \mathrm{D}^{\mathrm{b}}\right.$ lane 2$)$, anti-Qa- $1^{\mathrm{b}}$ (lane 32), anti-Qa-2 (lane 4) and R1.9.6 (lane 5). Other conditions as for Figure 3 
beled for 5 min with ${ }^{35} \mathrm{~S}$-methionine and the microsomal vesicles were then isolated and subjected to treatment with sodium carbonate at $\mathrm{pH} 11.5$ (Fujiki et al. 1982). This procedure opens sealed microsomal vesicles, thereby releasing soluble proteins, but lipid-associated molecules generally remain membrane bound. In Figure 5a the carbonate treatment is shown to partially separate $\mathrm{H}-2$ and Q4 molecules. The entire $\mathrm{H}-2$ remains membraneassociated under these conditions, while a major part of the Q4 is released into the supernate. The complete release of Q4, however, was never observed. This experiment reveals that most of the newly-synthesized Q4 molecules are water soluble under these conditions, but does not rule out the possibility that at physiological $\mathrm{pH}, \mathrm{Q} 4$ molecules may associate with the plasma membrane. To test this possibility, C57BL/ 6 spleen cells were surface-labeled with ${ }^{125} \mathrm{I}$ using the lactoperoxidase method (Robinson and Schirrmacher 1979). As shown in Figure 5b, lysates of surface-labeled cells contained $\mathrm{H}-2 \mathrm{~K}^{\mathrm{b}}, \mathrm{H}-2 \mathrm{D}^{\mathrm{b}}$, Qa-2, and Qa-1 molecules, but no Q4 material could be detected with $\mathrm{R} 1$.9.6. These results strongly suggest that $\mathrm{Q} 4$ is a soluble class I molecule which is not present on the cell surface.

Further properties of $Q 4$ polypeptides. The nucleotide sequence of $Q 4$ predicts potential sites of $\mathrm{N}$-glycosylation at amino acid positions 86 and 256. Partial digestion of Q4 molecules with endoglycosidase $F$ gives rise to two additional smaller forms, indicating the presence of two $\mathrm{N}$-linked glycans (data not shown). Estimation of the molecular weight of deglycosylated Q4 by SDS gel electrophoresis gives a value of $\mathrm{M}_{\mathrm{r}} 37000 \pm 500$. This is significantly larger than the value of 34684 determined from the protein sequence. This discrepancy cannot be attributed to anomalous behavior of class I molecules on SDS polyacrylamide gels, since deglycosylated $\mathrm{H}-2 \mathrm{D}^{\mathrm{b}}$ migrates according to its calculated molecular weight of 38 406. The apparent discrepancy for Q4 may be explained by unorthodox processing or modification of the polypeptide.

A limited enhancement of Q4 expression (approximately twofold) was achieved by treating the transfectants for $72 \mathrm{~h}$ with 20 units $/ \mathrm{ml}$ recombinant gamma interferon. This is consistent with the presence of an intact interferon consensus sequence in the $5^{\prime}$ flanking region of the $Q 4$ gene.

Similarity between $Q 4$ and $Q b-l$. The $Q b-1$ polypeptide was originally detected in lysates of ${ }^{35} \mathrm{~S}$-methionine labeled spleen cells using anti- $\beta_{2}$-microglobulin antibodies. Two allelic forms, $\mathrm{Qb}-1^{\mathrm{a}}$ and $\mathrm{Qb}-\mathrm{1}^{\mathrm{b}}$, are distinguishable by $2 \mathrm{D}$ gel electrophoresis. It was found that the $\mathrm{Q} 4$ molecule, isolated from $\mathrm{C} 57 \mathrm{BL} / 6$ spleen cells using R1.9.6, co-migrates precisely with $Q b-1^{b}$ (data not shown). A $Q 4$ allele was isolated from an AKR (Qb-1 ${ }^{\mathrm{a}}$ ) cosmid library. When expressed in $3 \mathrm{~T} 3$ cells, this gene was found to encode $\mathrm{a} \mathrm{Qb}-1$ molecule. This result supports the notion that $\mathrm{Qb}-1$ is encoded by the $Q 4$ gene.

\section{Discussion}

We chose to determine the nucleotide sequence of $Q 4$ because of increasing evidence that this gene is a particularly active contributor to gene conversion events within the mouse major histocompatibility complex. Transfer of sequence information by $Q 4$ has been shown by sequencing $H-2 K$ alleles from the $H-2 K^{b m}$ mutant mice of the bg series (Geliebter et al. 1986, Nathenson et al. 1986). The high frequency with which $Q 4$ acts as a donor gene may be attributed to a high degree of sequence homology between $Q 4$ and $H-2 K^{b}$, which could promote such exchange events. It has been suggested that the $H-2 K$ region may have been generated by duplication and translocation of a pair of $Q$ genes (Weiss et al. 1984). Thus, in C57BL/10 mice, $H-2 K^{b}$ would be the homolog of an even-numbered $Q$ gene, for example $Q 4, Q 6, Q 8$, or $Q 10$. The strong overall homology between $Q 4$ and $H-2 K^{b}$ suggests an ancient relationship between these genes. The fact that several stretches of strong sequence homology are located in regions of allelic variability may be explained by gene conversion. The fact that the $5^{\prime}$ region of Q4 is far more similar to $H-2$ genes than to $Q 5-Q 10$ may explain the observation that the R1.9.6 antibody binds several $\mathrm{H}-2$ molecules and $Q 4$ but is non-reactive with $Q a-2$ or other $Q$ gene products.

We have used a gene transfer approach to show that $Q 4$ encodes a secreted class I polypeptide. A similar polypeptide was found in C57BL/6 spleen cells. Secretion of the Q4 molecule can be explained by the presence of a translational stop signal in exon 5, which results in a protein with an incomplete membrane spanning segment. Similar defects are found in all the even-numbered $Q$ genes so far sequenced, namely $Q 4, Q 8$, and $Q 10$. The same single base deletion is found in $Q 4$ and $Q 8$, but in $Q 10,13$ bases are deleted suggesting that this defect has an independent origin. The Q10 product is secreted by the liver (Lew et al. 1986).

The general properties of the Q4 polypeptide are as predicted by the Q4 nucleotide sequence, except that the apparent molecular weight of the deglycosylated chain is about 3000 daltons greater than expected. Inspection of the Q4 nucleotide sequence does not allow an alternative exon-intron organization which would explain such a large discrepancy in the molecular weight. One possible explanation is that the signal peptide is not removed after synthesis. This would result in a polypeptide with a different $\mathrm{N}$-terminus and a calculated molecular weight of 36942 which is close to the value of 37000 estimated by gel electrophoresis.

Our data show that the Q4 molecule and Qb-1 are identical. This conclusion is supported by the fact that Q4 
molecules precipitated by R1.9.6 are indistinguishable from $\mathrm{Qb}-1$ by two-dimensional gel electrophoresis. Furthermore, Q4 molecules from spleen cells and from transfected fibroblasts are secreted together with $\beta_{2}$-microglobulin. It may be significant that the $Q a$ region encodes at least three secreted class I molecules. It is therefore possible that the $Q$ genes, in addition to being important for gene conversion, may also encode molecules of biological importance.

Acknowledgments. The authors thank Margot Lang for help with sequencing and Karin Reimbold for performing the transfections. We are indebted to Peter Altevogt and Ivan Lefkovits for 2D gel analyses, and to Jan Geliebter and Sarah Spencer for helpful comments. This work was supported by grants We1069 and Ro692 from the Deutsche Forschungsgemeinschaft.

\section{References}

Arnold, B., Burgert, H. G., Archibald, A. L., and Kvist, S.: Complete nucleotide sequence of the murine $H-2 K^{k}$ gene. Comparison of three $H-2 K$ locus alleles. Nucleic Acids Res. 12: 9472-9487, 1984

Devlin, J. J., Weiss, E. H., Paulson, M., and Flavell, R. A.: Duplicated gene pairs and alleles of class I genes in the $Q a-2$ region of the murine Mhc: a comparison. EMBO J. 4: 3203-3207, 1985

Dobberstein, B., Garoff, H., Warren, G., and Robinson, P. J.: Cell free translation and membrane insertion of the $\mathrm{H}-2 \mathrm{D}^{\mathrm{d}}$ histocompatibility antigen and $\beta_{2}$ microglobulin. Cell 17: 759-769, 1979

Elder, J. H. and Alexander, S.: Endo- $\beta$-N-acetylglucosaminidase F: Endoglycosidase from flavobacterium meningosepticum that cleaves both high mannose and complex glycoproteins. Proc. Natl. Acad. Sci. U.S.A. 79: 4540-4554, 1982

Flaherty, L.: Tla region antigens. In M.E. Dorf (ed.): The Role of the Major Histocompatibility Complex in Immunology, pp. 33-57, Garland STPM Press, New York, 1981

Fujiki, Y., Hubbard, A. L., Fowler, S., and Lazarow, P. B.: Isolation of intracellular membranes by means of sodium carbonate treatment. Application to endoplasmic reticulum. J. Cell Biol. 93: 97-102, 1982

Geliebter, J., Zeff, R. A., Schulze, D. H., Pease, L. R., Weiss, E. H., Mellor, A. L., and Nathenson, S. G.: Interaction between $K^{b}$ and Q4 gene sequences generates the $K^{t m 1}$ mutation. Mol. Cell. Biol. 6: $645-652,1986$

Harris, R.A., Hogarth, P.M., Penington, D. G., and McKenzie, I. F. C.: Qa antigens and their differential distribution on lymphoid, myeloid and stem cells. J. Immunogenet. 11: 265-281, 1984

Jaulin, C., Perrin, A., Abastado, J. P., Dumas, B., Papamatheakis, J., and Kourilsky, P.: Polymorphism in mouse and human class I $H-2$ and $H L A$ genes is not the result of random independent point mutations. Immunogenetics 22: 453-470, 1985

Kalb, V.F., Glasser, S., King, D., and Lingrel, J. B.: A cluster of repetitive elements within a 700 base pair region of the mouse genome. Nucleic Acid Res. 11: 2177-2184, 1983

Kimura, A., Israel, A., Le Bail, O., and Kourilsky, P.: Detailed analysis of the mouse $H-2 K^{b}$ promoter: enhancer-like sequences and their role in the regulation of class I gene expression. Cell 44: 261-272, 1986
Lew, A. M., Maloy, W. L., and Coligan, J. E.: Characteristics of the expression of the murine soluble class I molecule, Q10. J. Immunol. 136: $254-258,1986$

Maxam, A. M. and Gilbert, W.: Sequencing end-labeled DNA with base-specific chemical cleavages. Proc. Natl. Acad. Sci. U.S.A. 74: 560-564, 1977

Mellor, A. L., Weiss, E.H., Kress, M., Jay, G., and Flavell, R. A.: A non-polymorphic class I gene in the murine MHC. Cell 36 : 139-144, 1984

Mellor, A. L., Antoniou, J., and Robinson, P. J.: Structure and expression of genes encoding murine Qa-2 class I antigens. Proc. Natl. Acad. Sci. U.S.A. 82: 5920-5924, 1985

Michaelson, J., Flaherty, L., Bushkin, Y., and Yudkowitz, H.: Further biochemical data on Qa-2. Immunogenetics 14: 129-140, 1981

Nathenson, S. G., Geliebter, J., Pfaffenbach, G. M., and Zeff, R. A.: Murine major histocompatibility complex class I mutants: molecular analysis and structure-function implications. Annu. Rev. Im munol. 4: 471-502, 1986

Ploegh, H. L., Orr, H. T., and Strominger, J.: Major histocompatibility antigens: the human (HLA A, B, C) and murine (H-2K, H-2D) class I molecules. Cell 24: 287-299, 1981

Reyes, A. A., Schöld, M., and Wallace, B.: The complete amino acid sequence of the murine transplantation antigen $\mathrm{H}-2 \mathrm{D}^{\mathrm{b}}$ as deduced by molecular cloning. Immunogenetics 6: 1-9, 1982

Robinson, P. J.: Qb-1, a new class I polypeptide encoded by the $Q a$ region of the mouse $H-2$ complex. Immunogenetics 22 : 285-289, 1985

Robinson, P. J.: Two different pathways for the secretion of $Q a$ region associated class I antigens by mouse lymphocytes. Proc. Natl. Acad. Sci. U.S.A. 84: 527-531, 1987

Robinson, P. J. and Schirrmacher, V.: Differences in the expression of histocompatibility antigens on mouse lymphocytes and tumour cells: immunochemical studies. Eur. J. Immunol. 9: 61-66, 1979

Sanger, F., Coulson, A. R., Barrell, B. G., Smith, A. G. H., and Roe, B. A.: Cloning in single-stranded bacteriophage as an aid to rapid DNA sequencing. J. Mol. Biol. 143: 161-178, 1980

Soloski, M. J., Vernachio, J., Einhorn, G., and Lattimore, A.: $Q a$ gene expression: biosynthesis and secretion of Qa- 2 molecules in activated T cells. Proc. Natl. Acad. Sci. U.S.A. 83: 2949-2953, 1986

Steinmetz, M., Moore, K. W., Frelinger, J. G., Sher, B., Shen, F. W., Boyse, E. A., and Hood, L.: A pseudogene homologous to mouse transplantation antigens: transplantation antigens are encoded by eight exons that correlate with protein domains. Cell 25: 683-692, 1981

Taylor Sher, B., Nairn, R., Coligan, J. E., and Hood, L. E.: DNA sequence of the mouse $H-2 D^{d}$ transplantation antigen gene. Proc. Natl. Acad. Sci. U.S.A. 82: 1175-1179, 1985

Weiss, E. H., Golden, L., Zakut, R., Mellor, A. L., Fahrner, K., Kvist, S., and Flavell, R. A.: The DNA sequence of the $H-2 K^{b}$ gene: evidence for gene conversion as a mechanism for the generation of polymorphism in histocompatibility antigens. $E M B O J .2$ : 453-462, 1983

Weiss, E. H., Golden, L., Fahrner, K., Mellor, A. L., Devlin, J. J., Bullman, H., Tiddens, H., Bud, H., and Flavell, R. A.: Organisation and evolution of the class I gene family in the major histocompatibility complex of the C57BL/6 mouse. Nature 310: $650-655$, 1984

Wigler, M. S., Silverstein, L. S., Lee, A., Pellicer, Y., Cheng, T., and Axel, R.: Transfer of purified herpes virus thymidine kinase gene into cultured mouse cells. Cell 11: 223-232, 1977

Received June 15, 1987 